development and bone homeostasis. It has recently been demonstrated that RANK-activated astrocytes release CCL20 and attract $\mathrm{T}$ cells to the central nervous system in a model of Multiple Sclerosis and that transgenic RANK expression in the skin promotes aberrant epithelial cell proliferation and is sufficient to induce ectopic formation of tertiary lymphoid structures (TLS). Ductal epithelial cells (SGEs) have been implicated in Sjögren's Syndrome (SS) pathogenesis where they mediate immune recruitment by expression of pro-inflammatory chemokines and support the formation of pre-malignant myoepithelial lesions.

Objectives To address the role of RANK-RANKL interaction in primary (p) SS.

Methods A combination of human and mouse studies were used to explore the RANK-RANKL interaction in pSS. Salivary glands (SGs) and saliva samples from patients recruited in the OASIS cohort (University of Birmingham) were studied to evaluate this pathway in human disease. Consecutive stimulated saliva samples $(n=69)$ were analysed using Proseek Multiplex $\mathrm{INF}^{96} \times{ }^{96}$, covering 92 unique inflammation-related protein biomarkers. Taking advantage of a viral induced model of pSS we studied the effect of this pathway with a RANKL blocking antibody and by inducing gain of function with direct cannulation in the salivary glands of recombinant RANKL. Murine SGs were studied by immunofluorescence, flow cytometry and qPCR on total tissue and sorted cells.

Results Fourteen proteins in saliva were significantly separated between pSS and sicca controls, and elevated levels of just two proteins, RANKL and TNF $\beta$, could classify pSS or sicca with 75\% accuracy. Levels of salivary RANKL and CCL20 were strongly correlated $(\mathrm{r}=0.6 ; \mathrm{p}<0.01)$. We demonstrated that both human and murine inflamed SGEs upregulate both RANK and CCL20, a chemokine known to recruit pathogenic $\mathrm{T}$ cells. Upregulation of RANKL was found in human Th2 cells, classically associated with humoral responses and germinal centre (GC) formation. SGs from mice treated with antiRANKL antibody showed decreased epithelial proliferation, reduced $\mathrm{T}$ cell infiltration and defective TLS establishment. On the contrary, viral infected SGs treated with recombinant RANKL showed increased $\mathrm{T}$ cell infiltration, CCL20 expression and enhanced differentiation of GC B cells.

Conclusions In vivo RANK/RANKL interaction mediates recruitment of activated $\mathrm{T}$ cells that are skewed toward a Th2 phenotype. These, in turn, will favour the establishment of TLS in the SG. Those data were confirmed in human pSS, where expression of RANK is found in inflamed epithelium and RANKL detection in saliva is able to differentiate patients with pSS from sicca controls, thus candidates this pathway both for drug targeting and patient stratification.

Disclosure of interest None declared

\section{P018 PROTEASE ACTIVATED RECEPTOR 2 (PAR2) EXPRESSION IN THE MYELOID COMPARTMENT IMPACTS OSTEOCLASTOGENESIS}

${ }^{1} \mathrm{~S}$ McGrath*, ${ }^{2} \mathrm{~L}$ Hultin, ${ }^{3} \mathrm{JC}$ Lockhart, ${ }^{1} \mathrm{CS}$ Goodyear. 'Institute of Infection, Immunity, and Inflammation, University of Glasgow, Glasgow, UK; ${ }^{2}$ Respiratory, Inflammation and Autoimmunity, AstraZeneca, Mölndal, Sweden; ${ }^{3}$ Institute of Biomedical and Environmental Health Research, University of the West of Scotland, Paisley, UK

10.1136/annrheumdis-2018-EWRR2018.43

Introduction Protease activated receptor 2 (PAR2) is a G protein coupled receptor responsive to serine proteases, which plays a key role in inflammation and pain reception. Rheumatoid arthritis (RA) patients have up-regulated surface expression of PAR2 in circulating monocytes which correlates with disease activity. We have previously demonstrated that $\mathrm{Par}^{-/-}$ mice are protected from inflammation, bone erosion, and cartilage destruction in a Freund's Complete Adjuvant (FCA) induced arthritis model. However, it is unclear how PAR2 affects the composition of the myeloid compartment and osteoclastogenesis.

Objectives The aim of this study was to evaluate the impact of loss of PAR2 on the myeloid compartment and osteoclastogenesis.

Methods Bone marrow (BM) from 6-10 week old Par2 ${ }^{-/}$and control $\mathrm{C} 57 \mathrm{BL} / 6$ mice was cultured in pro-osteoclastogenic media for 5 days and the generated mature osteoclasts, tartrate-resistant acid phosphatase positive (TRAP) multinucleated cells, were counted. The resorption potential of these cells was assessed using osteolysis plates and the total osteo-resorption quantified after 7-14 days of culture. BM was also collected for flow cytometric analysis of the haematopoietic cellular composition (with the following markers: CD3, B220, CD11b, Ly6C, Ly6G, CD115, and CD117) and assessed for osteoclast precursors.

Results In vitro osteoclastogenesis revealed an increase in numbers of mature osteoclasts from Par $^{-/-}$BM compared to WT, corresponding with increased levels of resorption. Flow cytometry of BM from both WT and Par2 ${ }^{-/}$mice showed 3 distinct monocyte populations defined by the cell surface expression levels of CD11b and Ly6C. The overall ratio of these populations was not altered in $\operatorname{Par} 2^{-/-}$animals.

Conclusions BM from $\mathrm{Par}^{-/-}$mice has increased osteoclastogenic potential and overall resorptive activity. We propose that this is not due to differences in bone marrow residing osteoclast pre-cursor numbers. This study indicates a potential role for PAR2 in osteoclast differentiation and bone remodelling. Acknowledgements The research was funded by Medical Research Scotland (MRS) and supported by Astrazeneca.

Disclosure of interest S. McGrath: None declared, L. Hultin Employee of: Astrazeneca, J. Lockhart: None declared, C. Goodyear Consultant for: AstraZeneca

\section{P019 ULTRASOUND DETECTED TENOSYNOVITIS AS A MARKER OF SUBCLINICAL INFLAMMATION PRIOR TO ARTHRITIS ONSET}

Y Kisten*, H Rezaei, E af Klint, G Fei, AH Hensvold, Al Catrina. Department of Medicine, Rheumatology Unit and Clinic of the Karolinska University Hospital, Karolinska Institute, Stockholm, Sweden

\subsection{6/annrheumdis-2018-EWRR2018.44}

Introduction Prospective studies of individuals at increased risk of developing rheumatoid arthritis (RA) will further improve the understanding of disease development. Ultrasound emerges clinically useful in detecting subtle inflammatory changes in rheumatic diseases.

Objectives To investigate ultrasound (US) detected changes as markers for future arthritis development.

Methods Patients presenting with musculoskeletal complaints and a positive Anti-Citrullinated Protein Antibody (ACPA) test at primary care, were referred to Karolinska rheumatology clinic for further rheumatic joint disease assessments. Those lacking arthritis by clinical and US examination (defined as synovial hypertrophy with Doppler activity) were recruited 
into the Risk-RA clinical research program, and followed-up by our multidisciplinary rheumatology team. A total of 64 patients with complete US records were included between years $2015 \rightarrow 2016$. Our patient demographics were $84 \%$ (n 54/64) females, mean age 49 (range 22-82) years, median relative ACPA (times cut-off) 26 (range 1-174) titer, median visual analogue scale (VAS) pain 30, median VAS patient global 28 and median C-reactive protein was $1(0-20)$ at inclusion. Hand (Wrists, MCP's, PIP's, DIP's) and feet joints were USevaluated for synovial hypertrophy, hyperemia and bone erosions. The presence of wrist (compartments 1-6) and finger (flexor and extensor) tenosynovitis were assessed. Data from inclusion $\rightarrow$ follow-up visits until September 2017 were analysed. SPSS software version 25 was used (Univariate, Chisquare, T-test and Mann Whitney U-test) for comparisons.

Results At inclusion, none of the 64 patients had any signs of active joint inflammation. However, ultrasound changes for tenosynovitis were seen in 7 out of 64 patients, 3 of who also presented with mild hypertrophy without Doppler activity and one patient with mild Doppler hyperemia (without hypertrophy), and none with bone erosions at inclusion. Among all tendons evaluated bilaterally, tenosynovitis of the Extensor Carpi Ulnaris (ECU) wrist tendons (4 of 7 patients) and the 2nd finger flexor-tendons (3 of 7 patients) were most commonly affected.

Of the 57 patients without US-tendon changes, one had mild Doppler hyperemia (without hypertrophy). Patients with US-tendon changes were $86 \%$ (n 6/7) females, had mean age 56 years, median VAS pain 42, median VAS global health 20, mean $2.7 \mathrm{mg} / \mathrm{L}$ CRP, median relative ACPA titer 70 in comparison to patients without US-tendon changes, 75\% females, mean age of 48 years, median VAS pain 24, median VAS global health 28 , mean $2.7 \mathrm{mg} / \mathrm{L}$ CRP and median ACPA titer of 23. The numerical difference in pain and relative ACPA titer were non-significant $(p>0.05)$.

After follow up for mean 18 months (range 1-18), 7 out of $7(100 \%)$ with US-tendon changes at inclusion and 18 out of $57(32 \%)$ without US-tendon changes developed arthritis. Patients with US-tendon changes compared to those without tenosynovitis at inclusion, developed arthritis within 12 and 11 mean months follow-up, respectively.

Conclusions Our study shows that tenosynovitis is a specific marker for arthritis development in ACPA-positive patients with musculoskeletal symptoms. The role of inflammatory spreading from tendons (synovial sheath) to synovial tissue within joints need to be further investigated.

Disclosure of interest None declared

\section{\begin{tabular}{|l|l|l|}
\hline P020 & TOLL-LIKE RECEPTOR 9 INFLUENCES INFLAMMATORY
\end{tabular} ARTHRITIS AND OSTEOCLASTOGENESIS}

\footnotetext{
${ }^{1} \mathrm{~A}$ Fischer* ${ }^{2,3}$ S Abdollahi-Rodsaz, ${ }^{4} \mathrm{ACY}$ Yau, ${ }^{4} \mathrm{E}$ Lonnblom, ${ }^{4} \mathrm{R}$ Holmdahl, ${ }^{1} \mathrm{G}$ Steiner. ${ }^{1}$ Division of Rheumtology, Internal Medicine III, Medical University of Vienna, Vienna, Austria; ${ }^{2}$ Department of Rheumatology, Radboud University Nijmegen Medical Centre, Nijmegen, Netherlands; ${ }^{3}$ Division of Rheumatology, Department of Medicine, New York University School of Medicine, New York, USA; ${ }^{4}$ Medical Inflammation Research, Department of Medical Biochemistry and Biophysics, Karolinska Institute, Stockholm, Sweden
}

\subsection{6/annrheumdis-2018-EWRR2018.45}

Introduction Release and insufficient removal of endogenous nucleic acids may be involved in triggering autoimmune reactions important in the initiation of systemic autoimmune diseases including rheumatoid arthritis (RA). Nucleic acid sensing molecules, such as the endosomal Toll-like receptors (TLRs) 7 and 9, have been linked to pathogenic autoimmune processes, but their role in RA is less clear.

Objectives To gain more insight into the role of TLR9 in autoimmune arthritis, TLR9 inhibition was investigated in rats with pristane-induced arthritis (PIA). To further investigate TLR9 involvement, streptococcal cell wall (SCW) arthritis was induced in $\mathrm{TLR}^{-/-}$mice.

Methods Arthritis was induced in mice with SCW lysates and in rats with the mineral oil pristane. Rats were treated with a TLR9 antagonist, starting before disease induction. Arthritis was scored using established scoring systems, inflammation and bone erosion was quantified by histological analysis of the paws. Levels of $\alpha-1$-acid-glycoprotein (AGP), rheumatoid factor (RF) and IL-6 in sera were analysed. The role of TLR9 in osteoclast differentiation was investigated in vitro.

Results In PIA, which is T cell-dependent, the TLR9 antagonist reduced arthritis severity by $\sim 50 \%$. This was accompanied by a reduction of AGP, IL- 6 and RF in the sera of these animals. In addition, TLR9 inhibition led to reduced inflammation, bone erosion and cartilage degradation in the paws. Moreover, the $\mathrm{T}$ cell-dependent chronic phase of SCW arthritis was significantly suppressed in TLR9 ${ }^{-/}$mice. Remarkably, TLR7 and TLR9 mRNA levels strongly differed in the course of in vitro osteoclastogenesis. Whereas TLR7 expression did not change throughout osteoclastogenesis, expression of TLR9 was higher in precursor cells than in mature osteoclasts and stimulation with a TLR9 agonist (CpG) completely inhibited osteoclastogenesis.

Conclusions The results suggest a crucial role for TLR9 in the $\mathrm{T}$ cell-dependent phases of PIA and SCW arthritis and thus an important involvement of the DNA $(\mathrm{CpG})$ recognising TLR9 in the induction of arthritogenic autoimmune reactions. In addition, TLR9 also seems to play a role in the initiation of osteoclast differentiation which needs to be further elucidated in future experiments.

Disclosure of interest None declared

\section{P021 MEMBRANE TNF EXPRESSION ON MONOCYTES AND DIFFERENTIATION OF MONOCYTES INTO M2-M1 MACROPHAGES: 2 NEW BIOMARKERS OF RHEUMATOID ARTHRITIS}

${ }^{1} \mathrm{~A}$ Paoletti ${ }^{*},{ }^{2} \mathrm{G}$ Nocturne, ${ }^{2} \mathrm{~S}$ Bitoun, ${ }^{1} \mathrm{E}$ Riviere, ${ }^{1} \mathrm{~J}$ Pascaud, ${ }^{1} \mathrm{~B}$ Ly, ${ }^{2} \mathrm{X}$ Mariette. ${ }^{1}$ Rheumatology, U1184 INSERM IMVA; ${ }^{2}$ Rheumatology, Hopitaux Universitaire Paris Sud AP-HP, Le Kremlin Bicetre, France

10.1136/annrheumdis-2018-EWRR2018.46

Introduction Three monocyte subsets have been described based on their CD14 and CD16 expression profiles, the subpopulation $\mathrm{CD} 14^{+} \mathrm{CD} 16^{+}$being expanded in rheumatoid arthritis (RA) patients. Macrophages contribute in situ to the RA pathogenesis. They can display various states of activation or «polarisation ». Two distinct states of polarisation for macrophages have been recognised: the 'classically activated macrophage phenotype' (M1) and the 'alternatively activated macrophage phenotype' (M2). To sum-up, M1 are considered to be pro-inflammatory and $\mathrm{M} 2$ to be regulatory and antiinflammatory.

Objectives Here, we have assessed monocytes subsets and their capacity of differentiation into M2 or M1 macrophages in RA patients and controls. 